\title{
Automation of the design of the cross-section of the manipulator arms profile
}

\author{
Milan Mihola ${ }^{1}$, Zdenek Zeman², David Fojtik ${ }^{3}$ \\ ${ }^{1,2}$ Department of Robotics, VSB-Technical University of Ostrava, Czech Republic \\ ${ }^{3}$ Department of Control Systems and Instrumentation, VSB-Technical University of Ostrava, Czech Republic
}

\begin{tabular}{l} 
Article Info \\
\hline Article history: \\
Received Feb 15, 2021 \\
Revised Apr 9, 2021 \\
Accepted May 16, 2021 \\
\hline
\end{tabular}

Keywords:

Arm

Automation

Cross-section

Robot

RobotArmDesign

\begin{abstract}
The design of the arms of industrial robots and manipulators is a demanding process both in terms of expertise and in terms of the time required. For these reasons, algorithms have been created, with the help of which it is possible to design cross-sections of individual arms of robots and manipulators not only from the point of view of maximum allowed deflection but also from the point of view of minimizing cross-sectional dimensions or minimizing the weight of arms. These algorithms were subsequently used in the development of the software tool RobotArmDesign, with the help of which it is possible to simplify and shorten the arm design process significantly. This tool also has a connection to the SolidWorks CAD system and its simulation tools through its API interface, making it possible to refine robot arms designs while maintaining significantly shorter design times than would be the case with commonly used procedures. This tool's capabilities were demonstrated in the design of a robot arm with an angular structure and five degrees of freedom.
\end{abstract}

This is an open access article under the CC BY-SA license.

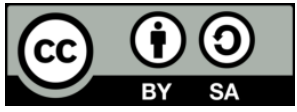

Corresponding Author:

Milan Mihola

Department of Robotics

VSB-Technical University of Ostrava

17. listopadu 2172/15, Ostrava 708 00, Czech Republic

Email: milan.mihola@vsb.cz

\section{INTRODUCTION}

Robotics is a multidisciplinary branch of technology. Scientific teams around the world are involved in the development of both complete robotic devices and their subsystems. From the point of view of industrial robotics, attention is currently paid mainly to the field of control, as described, for example, in the articles [1]-[3]. However, the possibility of using optimization algorithms in designing kinematic structures of industrial robots and manipulators, as described in the articles, also comes to the fore [4]-[6]. A somewhat neglected area is the possibility of automating the design process of both individual structural elements of robotic devices and entire mechanical systems. This is an area with significant potential in terms of creating better design proposals while significantly reducing the required design time of the equipment and the related improvement in work efficiency while reducing the costs associated with the actual design of the equipment.

Design of individual parts of industrial robots and manipulators is one of the most demanding processes, both in terms of the necessary expertise and time. For this reason, methodological procedures [7]-[9] and software tools [10]-[12] are being developed, which can significantly facilitate and speed up selected parts of this process. The development aims to transfer known methodological procedures and own experience gained in designing industrial robots, manipulators, and other types of mechatronic devices into a software tool that would automatically generate 3D models of devices or their individual parts. The development of such software tool is very demanding. Therefore, it was divided into several stages. This 
article describes the stage of automation of the cross-section design of the arms of industrial robots and manipulators.

Depending on the shape complexity of the arms, it is possible to design their cross-sections, resp. their dimensions, in various ways, e.g. according to the appropriate analytical method or with the help of the finite element method. The use of analytical methods is more advantageous for simpler arms, where it is possible based on known relationships (e.g. according to Betti's theorem or its modification, according to Maxwell's theorem, according to the method of integration of the differential equation of bending line. Panditta IK in [13]-[18] and required size of deflection, to design suitable cross-sections of the arms, resp. their dimensions. Analytical design methods are relatively fast. They also do not require specialized software tools, the acquisition of which is usually quite costly.

Finite element methods, which are used, for example, in strength analysis in many CAD systems, can also be used for simpler arms. Still, their advantages are mainly in more complex parts, with a more complicated distribution of loads, e.g. interaction of subsequent parts, where the procedures according to analytical methods would be too complex, or would not lead to the desired outcome. The disadvantages of this method of arms design include the need for specialized CAD systems and knowledge of working with them, sufficiently powerful computer equipment, and time. The strength analysis of one design variant of the proposed part can take tens of hours. For this reason, if possible and advantageous, it is advisable to consider a combination of these two approaches when designing the arms. The initial design of the arm profile and its dimensions would be carried out using a suitable analytical method, and only in the subsequent phase, the suitable CAD system would be used. In this way, it would be possible to significantly reduce the arm's total design time, thanks to the smaller number of required strength analyses.

Further reduction of design time can be achieved by automating this process. One possible way to do so is to use the application programming interface (API), which is often part of many CAD systems [19], [20]. In this case, the SolidWorks CAD system is used, with the help of which it is possible not only to create and subsequently analyze selected parts of the proposed equipment but also to perform kinematic and dynamic analyses, the results of which are used in strength analyses.

Further reduction of design time can be achieved by automating this process. One possible way is to use an application programming interface (API), which is part of many CAD systems [19]-[21]. In this case, the SolidWorks CAD system is used, with the help of which it is possible not only to create 3D models [22], [23] Development of an approach for knowledge-based system for CAD modeling, but also to perform their kinematic and dynamic analyzes. The results from these analyzes can then be used in strength analyzes or topological optimizations.

\section{RESEARCH METHOD}

Suppose an industrial robot or manipulator is designed for a specific manipulation or technological task. In that case, it is possible to specify the trajectory of the manipulation object (OM) movement or specify a position of the selected point of the technological effector, resp. determine the rotation of the effector, relative to this trajectory over time. The area or areas in which the proposed device could be placed relative to the trajectory should also be specified. Based on this information, it is then possible to design a suitable kinematic structure of an industrial robot or manipulator and its basic dimensions. With repeated kinematic and dynamic analyses, it is then possible to gradually design individual parts of the device.

The design process of the arms of industrial robots and manipulators is divided into two main parts. A preliminary design of suitable types of cross-sections and their dimensions is performed using a selected analytical method in the first part. In the second part, the design is refined using the module for strength analysis of CAD system SolidWorks. In both parts of the design process, an iterative process is used, where the shapes and dimensions of the cross-sections of the designed arms are gradually adjusted until the required values of their deflections are reached.

\subsection{Preliminary design of the arm profile}

By using known analytical methods, it is possible to determine the angle of rotation and deflection of an arm loaded with various combinations of forces, bending moments, torques and other types of loads. This process's complexity depends on the number and location of supports, respectively embeddings, the combination and places of action of individual loads, the cross-sectional shape of the proposed arm.

Within the preliminary design of the arms, the calculation procedure, according to the Castiglian theorem, is used, which is based on the deformation energies [17], [18]. To calculate the angle of rotation $\varphi$ (rad) and deflection $w(\mathrm{~mm})$ according to this theorem, relations (1) and (2) apply.

$$
\varphi=\frac{1}{E \cdot I} \int_{L} M(x) \cdot \frac{\partial M(x)}{\partial M} \cdot d x
$$




$$
w_{b}=\frac{1}{E \cdot I} \int_{L} M(x) \cdot \frac{\partial M(x)}{\partial F} \cdot d x
$$

where $E(\mathrm{MPa})$ is the modulus of elasticity in tension, $I\left(\mathrm{~mm}^{4}\right)$ is the moment of inertia of the cross-section, $M(x)(\mathrm{N} \cdot \mathrm{mm})$ is the moment, $\partial M(x) / \partial M$ is the partial derivative of the moment according to the moment acting in the calculated point, $\partial M(x) / \partial F$ is the partial derivative of the moment according to the force acting at the calculated point, $L(\mathrm{~mm})$ is the length over which the integration takes place.

In the case of industrial robots and manipulators with an angular structure, which is the most frequently used kinematic structure of industrial robots today [24], it is possible to count individual arms in the preliminary design as embedded beams (taking into account the required stiffness of rotary joints of robots), loaded at its end by forces and moments from previous parts of the proposed device, resp. from the load caused by the self-weight of the arms. Figure 1 indicates the possible load of the embedded beam, resp. of the designed arm by force $F(\mathrm{~N})$, bending moment $T_{b}(\mathrm{~N} \cdot \mathrm{mm})$ and continuous load $q(\mathrm{~N} / \mathrm{mm})$. The magnitude of the moment $M(x)(\mathrm{N} \cdot \mathrm{mm})$ at the point of entanglement, based on which it is possible to determine the angle of rotation and deflection at the endpoint of the bending line of the beam, can then be calculated using (3).

$$
M(x)=F \cdot \sin (\gamma) \cdot L+T_{b}+q \cdot \frac{L^{2}}{2}
$$

The continuous load $q(\mathrm{~N} / \mathrm{mm})$ can represent the external action force and the dead weight of the designed arm. In the case of a statically loaded beam, with a constant cross-section along its entire length, the magnitude of the continuous load is based on the density of the beam material $\rho\left(\mathrm{kg} / \mathrm{m}^{3}\right)$, its cross-sectional area $S\left(\mathrm{~m}^{2}\right)$ and the gravitational acceleration $g\left(\mathrm{~m} / \mathrm{sec}^{2}\right)$. It can be calculated using (4).

$$
q=\frac{\rho \cdot S \cdot g}{1000}
$$

However, in the case of a preliminary design of the arm profiles, it is also necessary to consider the dynamic effects based on their weight. For this reason, the previous relationship was modified to a different form (5), which is calculated by the sum of all accelerations (or their components) at the selected location of the beam (in the middle of the continuous load, or its part, which would be calculated) and in the direction of the investigated deflection of the proposed arm.

$$
q=\frac{\rho \cdot S}{1000} \cdot \sum_{i=1}^{n} a_{i}
$$

In the design of the arm's cross-section and its dimensions, it is based primarily on the value of the maximum required allowable deflection. However, the calculated bending stress value is also compared with the allowable value for the selected arm material. The design algorithm, using an iterative process, during which the cross-sectional dimensions are gradually read from the knowledge base from the smallest to the largest, is shown in Figure 2.

a)

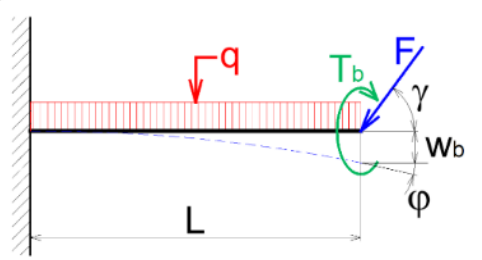

b) $\quad \mathrm{y}$

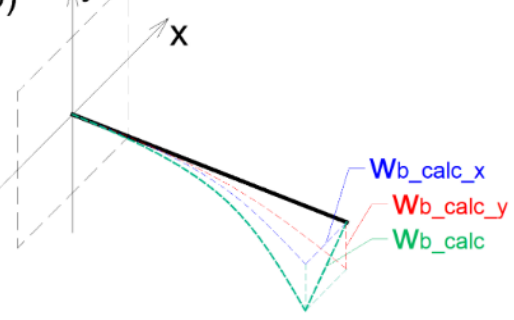

Figure 1. Embedded beam: (a) load on embedded beam, (b) deflections in individual planes and total spatial deflection 

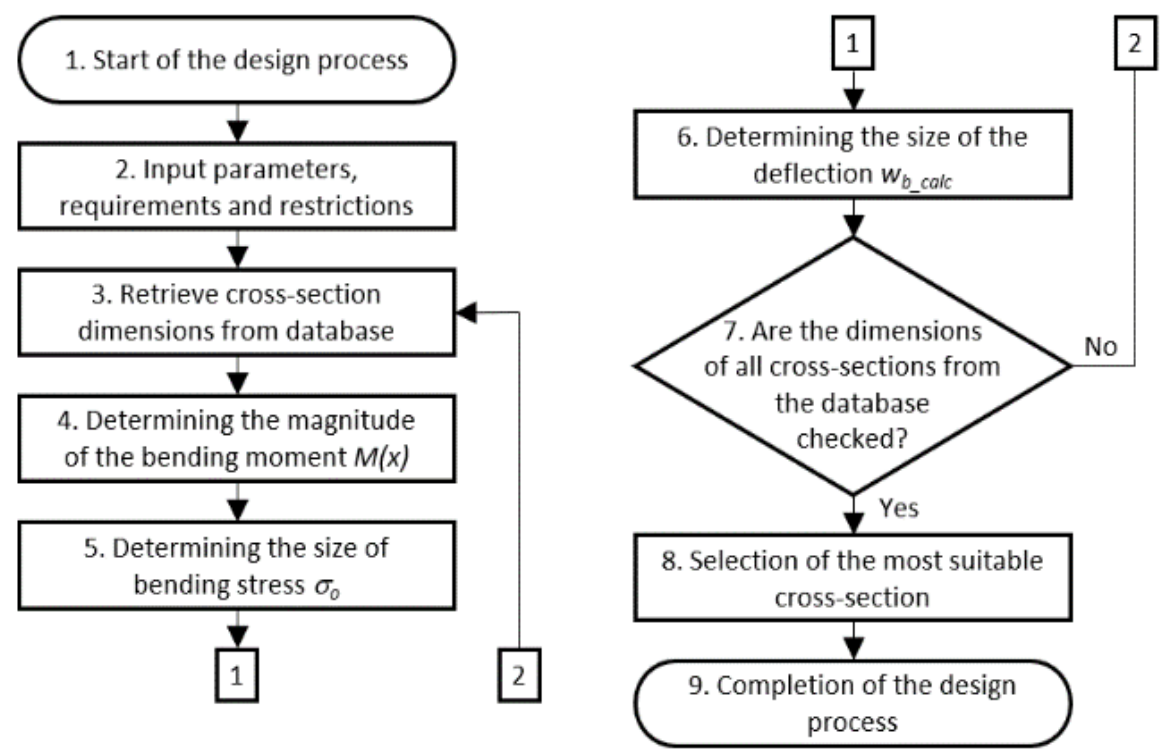

Figure 2. Algorithm for the preliminary design of arm cross-section dimensions

The design process is not completed when the arm's cross-sectional dimensions are found at which the calculated amount of deflection is smaller than required. Due to the effort to minimize the force effects on the following parts of the proposed device, cross-sections come to the forefront of the selection, with which it is possible to achieve the lowest possible weight of the arm. The maximum permissible external crosssectional dimensions, or other requirements and restrictions defined at the beginning of the preliminary design, can also play an important role. For this reason, deflection calculations are performed for all crosssections (or their dimensions) contained in the knowledge database. Only then is the final selection made based on other specified requirements and restrictions. In this way, it is possible to work on a more suitable type of cross-section and its dimensions than would be the case if the design were completed when the first cross-section of the arm was found in the database, with which it is possible to achieve less deflection than required.

The designed arm need not be subjected to a load in only one plane passing through its deflection line, as indicated in Figure 1(a). In such a case, it is necessary to distribute the force and moment effects into two suitably designed, mutually perpendicular planes passing through the beam's deflection line. The design of the beam cross-sectional dimensions would then proceed again according to the algorithm in Figure 2. However, the inspection of the maximum deflection would be performed in both mutually perpendicular planes. The total deflection $w_{b_{-} c a l c}(\mathrm{~mm})$ would be calculated using $(6)$, where $w_{b_{-} c a l c_{-} x}(\mathrm{~mm})$ and $w_{b_{-} c a l c_{-} y}$ $(\mathrm{mm})$ are the calculated partial deflections in individual, mutually perpendicular planes, see in Figure 1(b).

$$
w_{b_{-} c a l c}=\sqrt{w_{b_{-} c a l c_{-} x}^{2}+w_{b_{-} c a l c_{-} y}^{2}}
$$

The design of the arm cross-section would further proceed as described above. However, the value of $w_{b_{-} c a l c}$ would be compared with the value of the required maximum allowable deflection.

\subsection{Arm design using CAD software}

The arm of an industrial robot or manipulator with a pre-designed cross-section and its dimensions is then subjected to strength analysis in the CAD environment of the SolidWorks system. It is possible to perform strength analyses in two ways, as a separate element or as one of an assembly model's elements in motion analysis. In the first method, the arm is analyzed as a statically loaded embedded beam. In the second method, the proposed arm is analyzed within the entire motion cycle of the industrial robot or manipulator, within a selected part of this cycle, or at specified times of this cycle. Changes in applied loads during the movement cycle are reflected in the analysis here. This is a computationally demanding process and, therefore, more time-consuming than the arm's strength analysis as a separate element. In this article, only the first method will be considered. The arm design process algorithm is indicated in Figure 3, which, as in the case of the preliminary design, is an iterative process. 


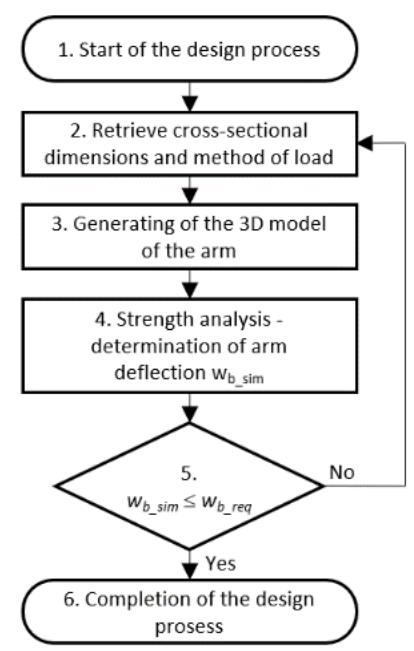

Figure 3. Algorithm of the arm profile design process using FEM

In the first iteration, a 3D model of the arm is created based on a preliminary design, which is then subjected to strength analysis, based on which the deflection of the arm $w_{b_{-}}$sim $(\mathrm{mm})$ is determined. If this value is less than the required deflection $w_{b_{-} r e q}(\mathrm{~mm})$ value, the following strength analysis will be subjected to an arm of smaller cross-section, resp. lower weight, which was no longer suitable in the preliminary design. If this arm does not comply, the design process will be completed with the most appropriate crosssection from the preliminary design. If this arm is also suitable, the same procedure will continue until a cross-section of dimensions is found, which would no longer be suitable from the required deflection point of view. The last, dimensionally suitable cross-section will be marked as the most suitable cross-section, and thus the design process will be completed.

Otherwise, if the arm based on the preliminary design dimensions does not meet the required deflection, the strength analysis will be subjected to the arm of a larger cross-section, resp. higher weights in the order specified in the preliminary design. If this arm is not suitable either, this procedure will be repeated until a dimensionally suitable cross-section is found in terms of the required deflection. That will complete the design process.

The time required for this process depends on the complexity of the analyzed 3D model, its size, the fineness of the mesh used in the given strength analysis and the number of iterations. However, at least two iterations will always be performed. The process of creating a 3D model of the arm, preparation and performance of strength analysis, and subsequent comparison of the achieved results with the required results, can be automated using the API of CAD system SolidWorks [19], [20].

\subsection{RobotArmDesign software tool}

To speed up and simplify the design of the arms of industrial robots and manipulators, the algorithms and calculation procedures mentioned above were converted into the software tool RobotArmDesign, see in Figure 4.

When designing an arm, you must first specify its length and the material from which it is to be made. Subsequently, the magnitudes of the loading forces and moments at the end of the arm and the acceleration at its centre of gravity are entered. Then it is possible to select either one of the available profiles of the proposed arm, or to allow selection from all available profiles stored in the database. Before starting the design using the "Preliminary proposal" button, it is necessary to enter the proposed arm's maximum permissible deflection value. Before starting the design, it is possible to check the arm's design options with minimum external dimensions or minimum weight. In the case of rectangular cross-sections, it is possible to enter restrictions regarding their height and width ratios.

When the preliminary design is complete, the RobotArmDesign software tool lists the matching profiles in the lower window, including their weight and deflection size. In the window below the "Preliminary proposal" button, the time of the preliminary design of the arm cross-section and the number of analyzed cross-sections are listed. The maximum pre-design time (on a computer configured with an AMD Ryzen 5 2600X processor, 16 GB of RAM, a graphics card equipped with an NVidia GeForce GTX 1650 chip, and a 500GB SSD) will not exceed 1 minute.

By using the "CAD design" button, it is possible to design the arm cross-section, including its strength analysis in the CAD environment of the SolidWorks system. However, in this case, it is necessary to 
limit the selection to only one specific type of cross-section, one explicit material and select one of the options "Minimum Dimensions" or "Minimum weight". After pressing the "CAD design" button, the RobotArmDesign software tool first proceeds according to the algorithm indicated in Figure 2. After performing the preliminary design, the procedure follows the algorithm indicated in Figure 3. An example of the strength analysis result is shown in Figure 5. Window of the RobotArmDesign software tool. In the window below the "CAD design" button, the design time of a suitable cross-section of the arm and the total number of analyzed profiles are then listed. The maximum design time depends on the number of strength analyzes performed. In most cases (up to five strength analyses performed) it does not exceed 10 minutes.

The "Create Excel" button can be used to export the results of the preliminary design of the arm cross-section to a pre-prepared MS Excel file and then view these results in graphs, Figure 6 showing the dependencies between the dimensions of available cross-sections in the database and deflections at a specified load. They also plot the relationship between the given cross-sections' dimensions and their weight, for a given arm length.

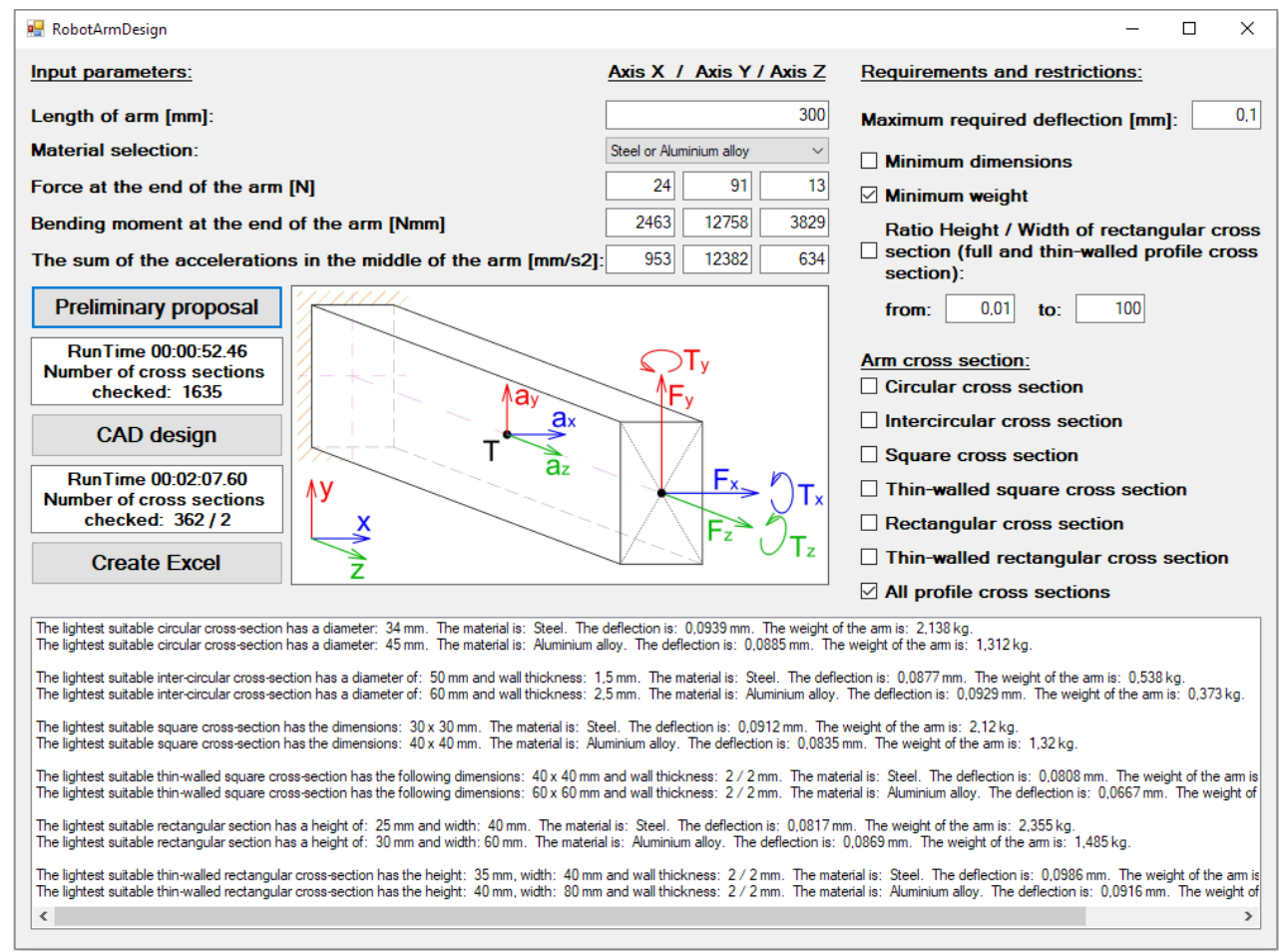

Figure 4. RobotArmDesign software tool

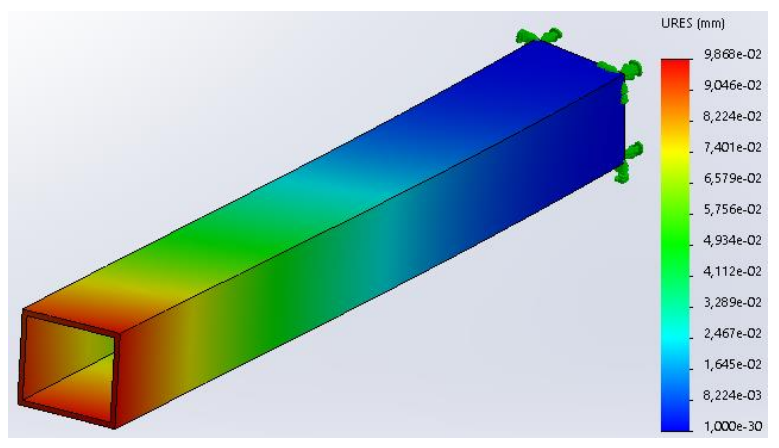

Figure 5. Result of strength analysis of the arm of a rectangular thin-walled cross-section
Proposal of arm of circular cross-section (material Steel)

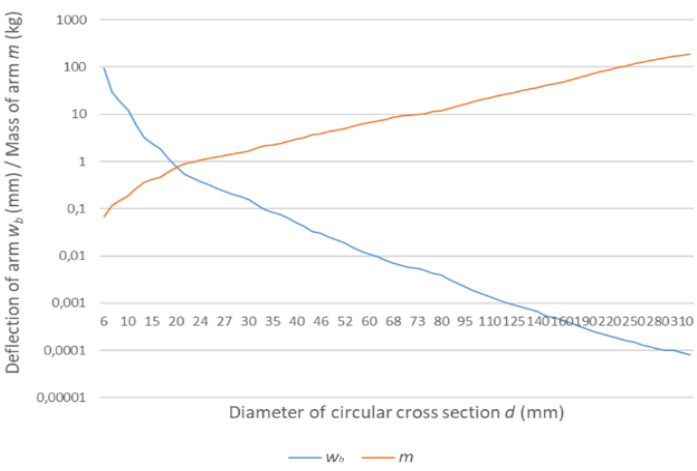

Figure 6. Dependence of the size of the deflection of the full circular cross-section arm and its weight for the specified load and all diameters listed in the profile database 


\section{RESULTS AND ANALYSIS}

Functionality and benefits of the algorithms mentioned above, respectively RobotArmDesign software tool was verified on the manipulation task indicated in Figure 7. A robot with an angular structure and 5 degrees of freedom was designed to manipulate a cylindrical object with a diameter of $52 \mathrm{~mm}$, a length of $200 \mathrm{~mm}$ and a weight of $3,313 \mathrm{~kg}$. The length of the trajectory is $1314,16 \mathrm{~mm}$, and OM travels it in 3 seconds.

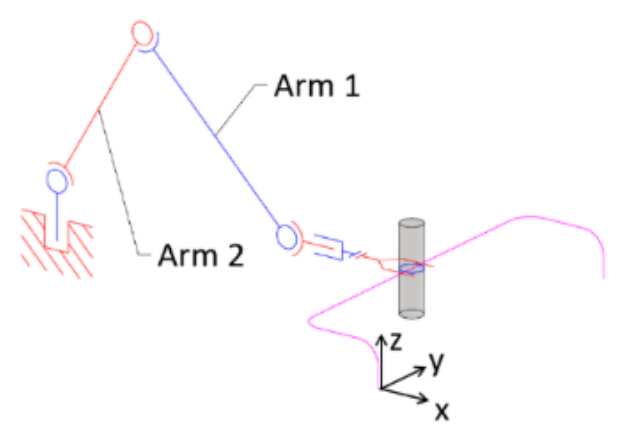

Figure 7. Design of the kinematic structure of the manipulator

Based on the robot's kinematic structure, the object of manipulation and repeated kinematic and dynamic analyzes in the CAD environment of the SolidWorks system, the individual elements of the robot were gradually designed. Starting with the end effector and ending with the robot base. The previously created Drive Picker software tool was used to design the Harmonic Drive units of the CanisDrive series. 3D models of structural elements related to power units were generated with the help of a previously created knowledge base. The following graphs (Figure 8) show the forces, moments and accelerations required to design Arm 1 arm, obtained from a dynamic analysis of the proposed device. The length of this arm is $300 \mathrm{~mm}$.

Table 1 shows preliminary dimensional designs for individual types of cross-sections (including the indication of the arm's final weight), for Steel and Aluminum alloy materials, at selected moments of OM movement. For each type of cross-section, material and time, two values are given, the minimum size of a suitable cross-section and the cross-section's size with the lowest achievable weight, for the required maximum deflection of $0,01 \mathrm{~mm}$. If a suitable cross-section is not found, there is a dash in the table column.
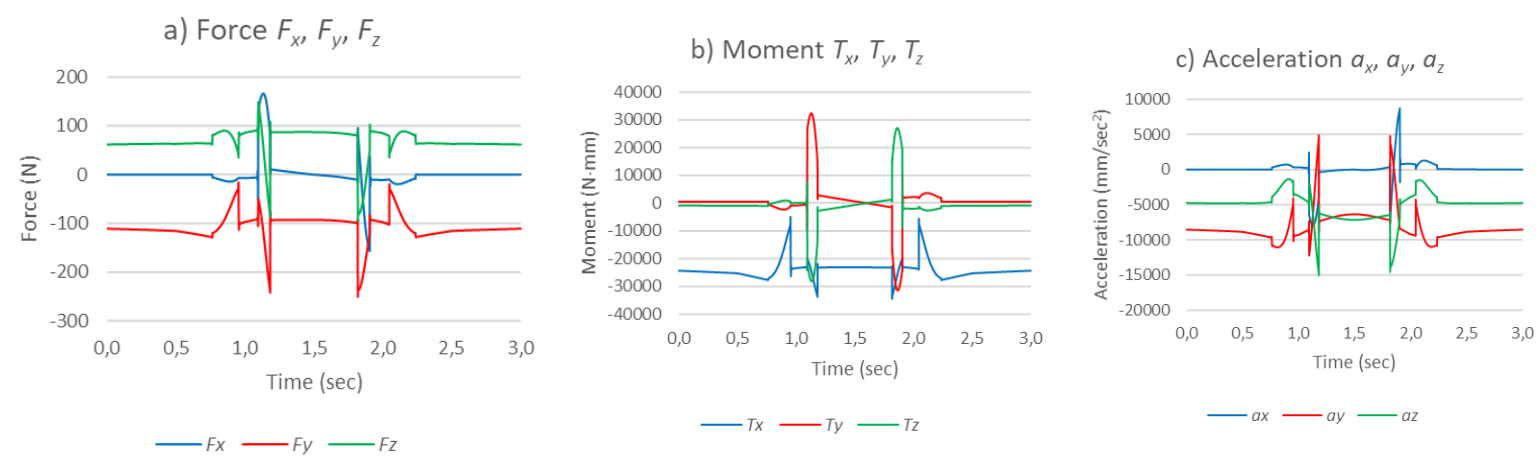

Figure 8. Graphs with courses: (a) forces $F_{x}, F_{y}, F_{z}$, (b) moments $T_{x}, T_{y}, T_{z}$, (c) accelerations $a_{x}, a_{y}, a_{z}$

In the second and third columns of Table 2, for each type of cross-section and materials, the crosssections' dimensions are given (again for the minimum size of a suitable cross-section and the cross-sectional size with the lowest achievable arm weight). It also shows the sizes of the calculated deflection and the number of sizes checked within the given type of cross-section. The fourth column shows the percentage difference in weight between the arms of a given type of cross-section with the smallest dimensions and the smallest weight. 
Table 1. Preliminary design of the dimensions of the cross-sections of the arms at selected moments of

OM movement

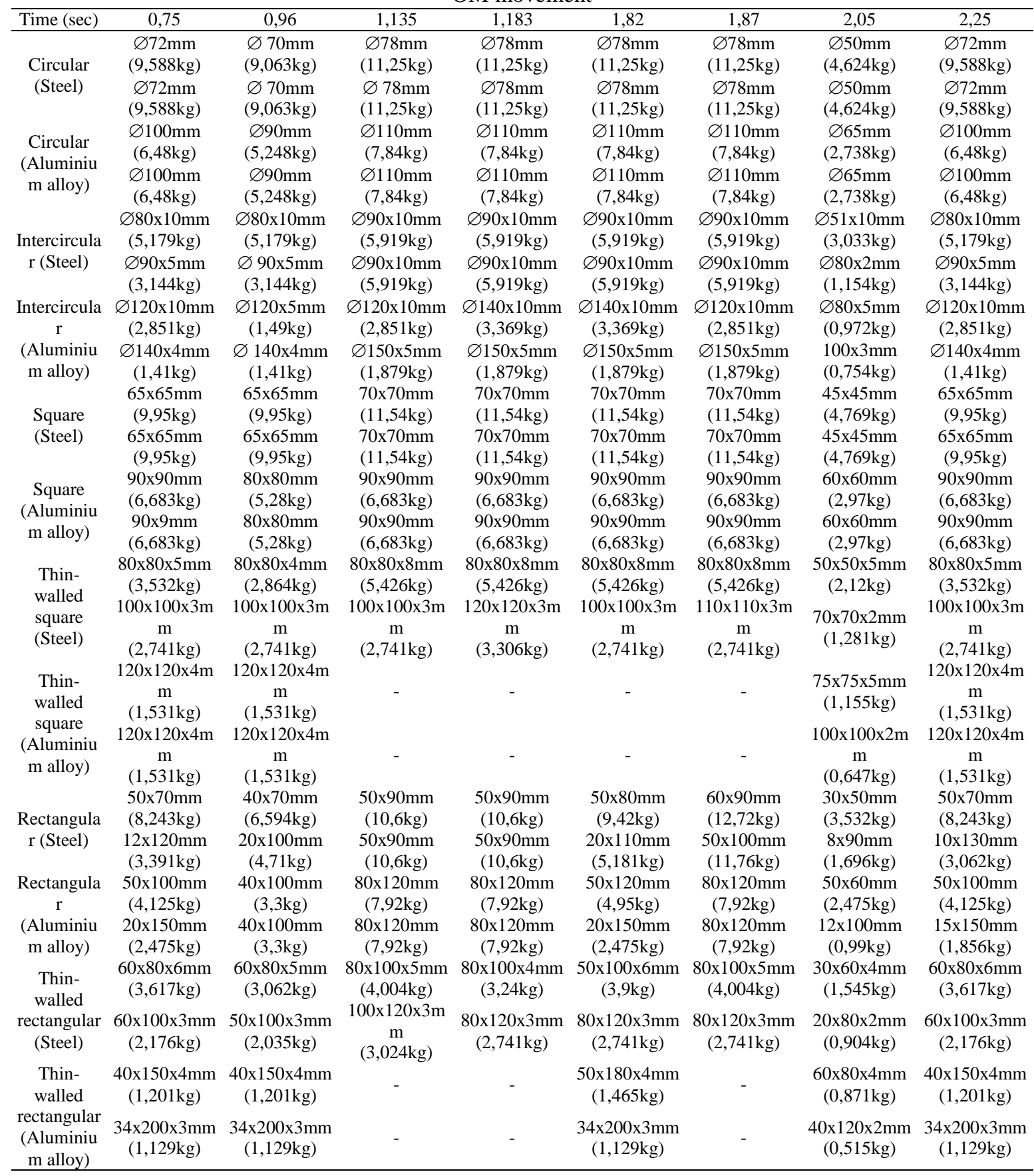

In the fifth and sixth columns of Table 2, the dimensions are given for each type of cross-section and material, again for the minimum size of a suitable cross-section (the size of the cross-section with the lowest achievable arm weight) designed using strength analyzes performed in SolidWorks CAD. Again, it shows the deflection sizes and the number of sizes checked within the given cross-section. However, there are two values, separated by a slash. The first indicates the number of sizes of a given cross-section, checked in the preliminary design. The second (after the slash) shows the number of strength analyzes performed to find a cross-section of suitable dimensions.

It is clear from Table 2 that for this particular case, it was necessary to perform only two strength analyses for most cross-sections. Overall, it could be concluded that it is possible to obtain a cross-section of the arm either dimensionally satisfactory or close to the preliminary design process's desired result. 
Table 2. Design of cross-sections using strength analysis and comparison with the results of preliminary design

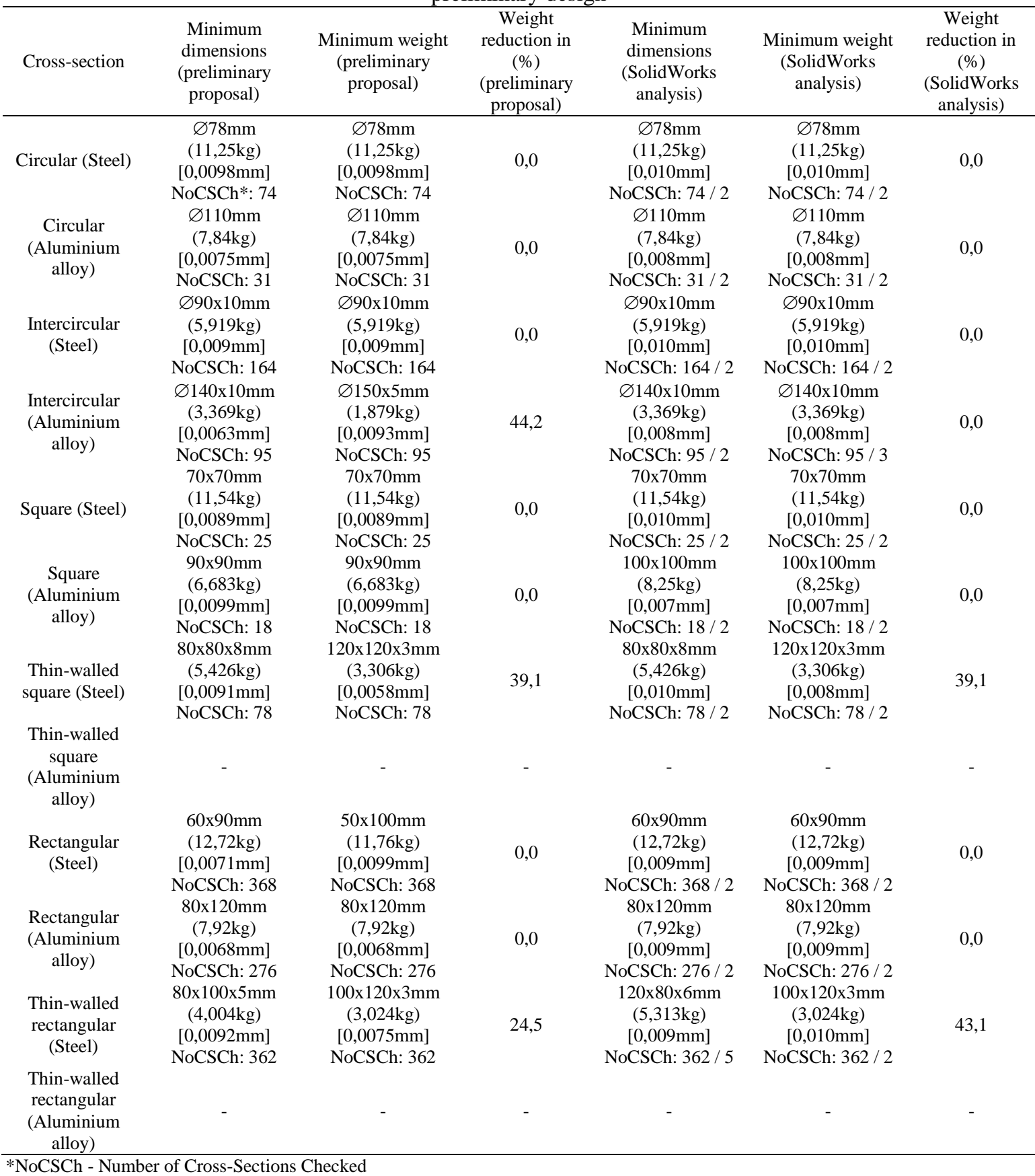

Figure 9 shows the structural designs of the Arm 1 arm according to the cross-sections in Table 2, including flanges whose connection dimensions correspond to the respective designed rotary joints of the robot (a) $\varnothing 78$, material Steel; b) $\varnothing 110$, Aluminum alloy material; c) $\varnothing 90 x 10$, material Steel; d) $\varnothing 140 x 10$, material Aluminum alloy; e) 70x70, material Steel; f) 100x100, material Aluminum alloy; g) 80x80x8, material Steel; h) 120x120x3, material Steel; i) 60x90, material Steel; j) 80x120, material Steel; k) 120x80x6, material Steel; 1) 100x120x3, material Steel.

If we compare the proposed cross-sections of the arms only from the point of view of the arm's weight, it is evident that the thin-walled ones perform significantly better than the solid ones. Aluminium alloy arms are also lighter than steel arms. But they also have larger external dimensions. The larger external dimensions of the proposed arm are not problematic from the point of view of the actual construction or the robot's planned deployment. It is more appropriate to use thin-walled cross-sections made of Aluminum alloy 
and thus achieve its lower weight. Otherwise, the use of Steel or full cross-sections is more advantageous (in the case of full cross-sections, there is significantly more space for possible subsequent topological optimization than thin-walled cross-sections).

Based on the achieved results, the Arm 1 arm was designed from a square thin-walled cross-section measuring $80 \times 80 \times 8 \mathrm{~mm}$, made of Steel. Its weight is $44,3 \%$ greater than in the case of using a rectangular thin-walled cross-section measuring 100x120x3 mm, which was the best in terms of weight. But from the point of view of the cross-section's external dimensions, this is a significantly more compact solution, better corresponding to the dimensions of the joints, resp. other parts of the proposed robot. Due to the excessive external dimensions, aluminium alloy profiles were not considered, despite the significantly lower achievable weights than the final solution.

Similarly, the Arm 2 arm was designed. A rectangular thin-walled cross-section with dimensions of 100x80x3 mm, made of Steel, was designed for this arm based on the achieved results. Figure 10 shows the resulting $3 \mathrm{D}$ model of the robot, including the OM and its trajectory.

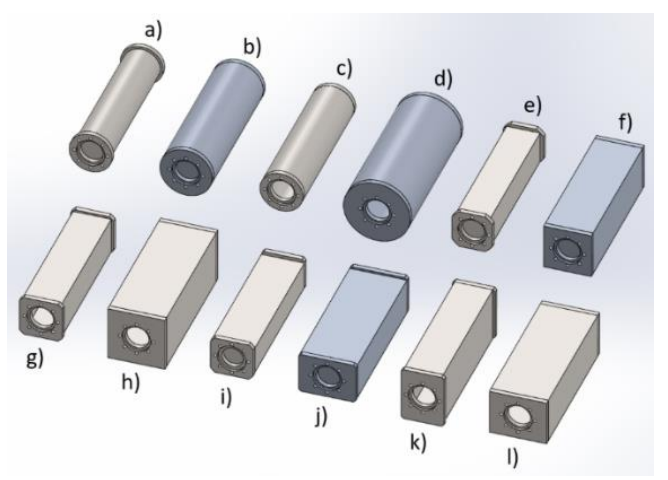

Figure 9. Arm 1 designs

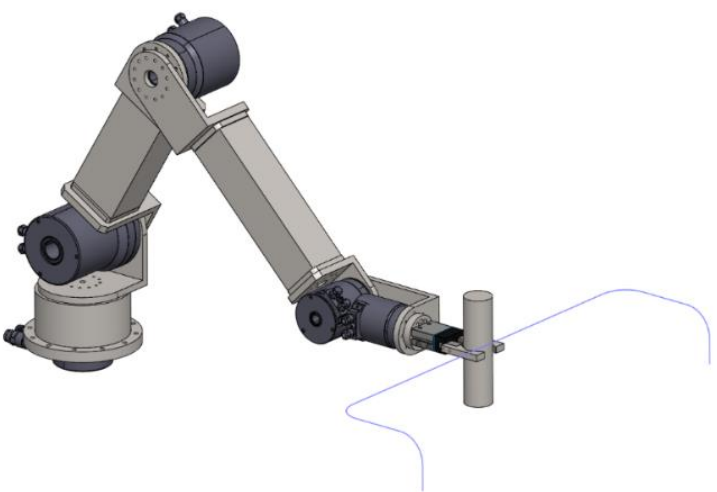

Figure 10. 3D model of the robot arm

At the time of writing, no information was available on a similar way of designing the arms of industrial robots and manipulators or similar structural elements. Commonly used design procedures, or available software tools, were designed to solve partial tasks within the described issues. By modifying them with regard to the design of the arms of industrial robots and manipulators, interconnection, the addition of other necessary algorithms, and subsequent creation of the software tool RobotArmDesign, enabling the design of a suitable cross-section of arms of robots and manipulators, can be considered unique. At the same time, not only was the required reduction of time and reduction of design complexity achieved but also the way was opened to automate the design process of complete arms of industrial robots and manipulators.

\section{CONCLUSION}

Algorithms for the preliminary design of arms of industrial robots and manipulators, resp. the RobotArmDesign software tool built on them has proven to be very beneficial both in terms of simplification and reducing design time. Instead of tens of minutes, or even tens of hours, spent trying to find the most suitable of the available cross-sections using commonly used procedures and available software tools, this process can be reduced to units of minutes. When designing the cross-section of the Arm 1 and Arm 2 arms, the possibility of using SolidWorks CAD simulation tools also proved to be very beneficial. In all cases, the preliminary design's cross-sections proved to be the most suitable, resp. in some cases, they did not meet the condition of the required maximum permissible deflection. All this without the developer working with these simulation tools or working with the SolidWorks CAD system in general.

\section{ACKNOWLEDGEMENTS}

This article was developed with the support of the project Research Centre of Advanced Mechatronic Systems, reg. no. CZ.02.1.01/0.0/0.0/16_019/0000867 in the frame of the Operational Program Research, Development and Education. 


\section{REFERENCES}

[1] TR. Prathab, RSM. Malar,and T. Ahilan, "A Method of Extended Jacobian and Firefly Algorithm for the Kinematic Analysis of Planar Robots," International Journal of Robotics and Automation (IJRA), vol. 6, no. 2, pp. 141-150, 2017, doi: 10.11591/ijra.v6i2.pp141-150.

[2] FJ. Castillo-Garcia, P. Rea, A. Gonzalez-Rodriguez, and E. Ottaviano, "On the Design of a 4 Degrees-of-freedom Pick and Place Cable Suspended Parallel Manipulator," International Journal of Robotics and Automation (IJRA), vol. 6, no. 4, pp. 286-302, 2017, doi: 10.11591/ijra.v6i4.pp286-302.

[3] A. Azarfar, "Self-tuning Fuzzy Task Space Controller for Puma 560 Robot," International Journal of Robotics and Automation (IJRA), vol. 7, no. 4, pp. 273-282, 2018, doi: 10.11591/ijra.v7i4.pp273-282.

[4] S. Kumar, K. Rani, VK. Banga, "Robotic Arm Movement Optimization Using Soft Computing," International Journal of Robotics and Automation (IJRA), vol. 6, no. 1, pp. 1-14, 2017, DOI: 10.11591/ijra.v6i1.pp1-14.

[5] J. Whitman and H. Choset, "Task-Specific Manipulator Design and Trajectory Synthesis," IEEE Robotics and Automation Letters, vol. 4, no. 2, pp. 301-308, April 2019, doi: 10.1109/LRA.2018.2890206.

[6] S. Ha, S. Coros, A. Alspach, J. M. Bern, J. Kim and K. Yamane, "Computational Design of Robotic Devices From High-Level Motion Specifications," IEEE Transactions on Robotics, vol. 34, no. 5, pp. 1240-1251, 2018, doi: 10.1109/TRO.2018.2830419.

[7] U. Lindemann, "Methodische Entwicklung technischer Produkte: Methoden flexibel und situationsgerecht anwenden," 3th ed. Berlin: Springer-Verlag, 2009, p. 341.

[8] VDI-Fachbereich Produktentwicklung und Mechatronik, Entwicklungsmethodik für mechatronische Systeme, Berlin: Beuth Verlag; 2004. 118 p.

[9] R. Isermann, "Mechatronic design approach," In: Bishop RH. The Mechatronics Handbook. 1 edition. Boca Raton: CRC Press; 2002. pp. 3.

[10] KISSsoft, Bubikon, Switzerland: KISSsoft AG, 2021, [Online]. Available: https://www.kisssoft.com/en.

[11] MITCalc, Decin, Czech Republic: MITCalc, 2021, [Online]. Available: https://mitcalc.com/.

[12] EJ. Reddy, CNV. Sridhar, V. Pandurangadu, "Research and development of knowledge based intelligent design system for bearings library construction using SolidWorks API," In Berretti S., Thampi S., Dasgupta S. (eds) Intelligent Systems Technologies and Applications. Advances in Intelligent Systems and Computing, vol 385. Springer, Cham; 2016. p. 311-319, doi: 10.1007/978-3-319-23258-4_27.

[13] IK. Panditta, "Deflection of Structures using Modified Betti's Theorem," International Journal of Aerospace Sciences, vol. 2, no. 1, pp. 11-13, 2013, doi: 10.5923/j.aerospace.20130201.02.

[14] IK. Panditta, MM. Wani, "Deflection of Structures using Principle of Quasi Work," International Journal of Aerospace Sciences, vol. 1, no. 5, pp. 85-91, 2012, doi: 10.5923/j.aerospace.20120105.01.

[15] JS. Przemieniecki, Theory of Matrix Structural Analysis, Revised edition. Dover: Dover Publications, 2012, 480 p.

[16] BS. Bedenik, CB. Besant, Analysis of Engineering Structures, Chichester: Horwood Publishing, 1999, p. 392.

[17] EJ. Hearn, Mechanics of Materials 1, 3th ed. Oxford: Butterworth-Heinemann, 1997, p. 450.

[18] KE. Kurrer, The History of the Theory of Structures: From Arch Analysis to Computational Mechanics, Berlin: Ernst \& Sohn, 2008, p. 848.

[19] T. Chen, XX. Yan, ZH. Yu, "The Research and Development of VB and Solidworks-Based 3D Fixture Component Library," Applied Mechanics and Materials, vol. 300-301, pp. 301-305, 2013, doi: 10.4028/www.scientific.net/amm.300-301.301.

[20] AC. Lad, AS. Rao, "Design and Drawing Automation Using Solid Works Application Programming Interface," International Journal of Emerging Engineering Research and Technology,vol. 2, no. 7, pp. 157-167, 2014.

[21] ZFZ. Abidin, MNO. Zahid, "Real time object customization in CAD system," Indonesian Journal of Electrical Engineering and Computer Science, vol. 15, no. 2, pp. 1066-1075, 2019, doi: 10.11591/ijeecs.v15.i2.pp1066-1075.

[22] EJ. Reddy, VP. Rangadu, "Development of knowledge based parametric CAD modelling system for spur gear: An approach," Alexandria Engineering Journal, vol. 57, no. 4, pp. 3139-3149, 2018, doi: 10.1016/j.aej.2018.07.010.

[23] EJ. Reddy, N. Venkatachalapathi, VP. Rangadu, "Development of an approach for Knowledge-Based System for CAD modelling," Materials Today: Proceedings, vol. 5, no. 5, pp. 13375-13382, 2018, doi:10.1016/j.matpr.2018.02.330.

[24] IFR International Federation of Robotics, “IFR," Frankfurt/Main, Germany: IFR, 2021, [Online]. Available: https://ifr.org/.

\section{BIOGRAPHIES OF AUTHORS}

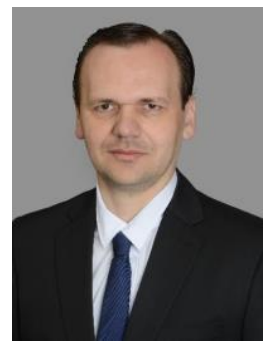

Milan Mihola received his M.Sc. degree, Ph.D. and defended his habilitation thesis at VŠB Technical University in Ostrava. He is currently an Associate professor and researcher at VŠB Technical University in Ostrava. His main research areas include robotics, mechatronics, and construction in the fields of production automation. 


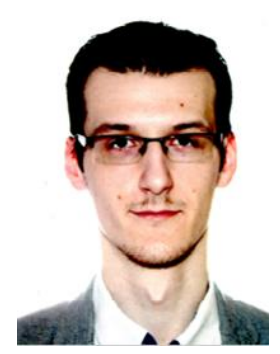

Zdenek Zeman received his M.S. degree in Robotics at VSB - Technical University of Ostrava. Zdenek Zeman is now working towards the Ph.D. degree in Robotics at the same university. His main research interests include automation of machine design processes and design of flexible $3 \mathrm{D}$ modeling methods.

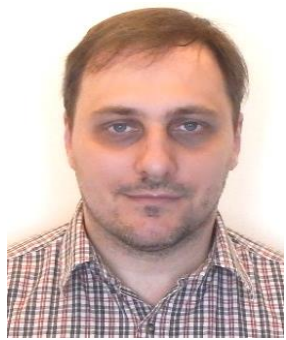

David Fojtik received his M.Sc. degree and Ph.D. at VSB - Technical University of Ostrava. Now he is an Assistant professor and senior researcher at VSB - Technical University of Ostrava. His main research directions include intelligent sensors and automatic diagnostic systems and real-time control systems. 